\title{
Salt and Pepper Noise Removal Using Resizable Window and Gaussian Estimation Function
}

\author{
Suhad A. Ali, C. Elaf A. Abbood, C. Shaymaa Abdul Kadhm
}

Babylon University, Science College for Women, Computer Department, Babylon, Iraq

\begin{tabular}{l} 
Article Info \\
\hline Article history: \\
Received Jun 26, 2016 \\
Revised Aug 7, 2016 \\
Accepted Aug 27, 2016 \\
\hline Keyword: \\
Gaussian function estimation \\
Max filter \\
Noise detection \\
PSNR and SSIM metrics \\
Resizable window \\
Salt and pepper noise
\end{tabular}

\begin{abstract}
Most types of the images are corrupted in many ways that because exposed to different types of noises. The corruptions happen during transmission from space to another, during storing or capturing. Image processing has various techniques to process the image. Before process the image, there is need to remove noise that corrupt the image and enhance it to be as near as to the original image. This paper proposed a new method to process a particular common type of noise. This method removes salt and pepper noise by using many techniques. First, detect the noisy pixel, then increasing the size of the pixel window depending on some criteria to be enough to estimate the results. To estimates the pixels of image, the Gaussian estimation function is used. The resulted image quality is measured by the statistical quantity measures that's Peak Signal-to-Noise Ratio (PSNR) and The Structural Similarity (SSIM) metrics. The results illustrate the quality of the enhanced image compared with the other traditional techniques. The slight gradual of SSIM metric described the performance of the proposed method with high increasing of noise levels.
\end{abstract}

Copyright (C) 2016 Institute of Advanced Engineering and Science. All rights reserved.

\section{Corresponding Author:}

C. Elaf A. Abbood

Computer Dep., Science College for Women

Babylon University

Babylon, Iraq

Email: wsci.elaf.ali@uobabylon.edu.iq

\section{INTRODUCTION}

Image noise can be catching the image from many different noise resources. These resources are containing bit errors in transmission, faulty pixels and damaged memory location. The noise types include Gaussian, Salt and pepper, Poisson and Speckle noise. Salt and pepper described as a white and black dotes distributed on the image in random way with a specific level of that noise [1]. There are many techniques are used to enhance the image that contaminates with salt and pepper noise and limiting the effect of it. Standard Median Filter (SMF) is one of the nonlinear filters that remove the salt and pepper noise by finding the median value of the filter window to be the predicted new pixel value. This filter has drawback with this type of noise that it is not get a good performance with a high level of that noise; furthermore, it removes necessary details in the image [2]. Consequently, the Weighted Median Filter (WMF) and Recursive Weighted Median Filter (RWMF) were introduced that used to increasing the quality of resulted image by get a high rate to the necessary pixels in the window that specified in a special weight [3]. But the quality of the resulted image stilled not appropriate because there is no predict if the pixel is corrupted or not. Therefore, noise detection techniques and decision based algorithms are proposed to increase the quality of the processed image like Adaptive Median Filter (AMF) and Progressive Switching Median filter (PSMF) were proposed. These filters are used noise detection techniques to find the noisy pixels then processed it using the standard median filter with a special conditions [4],[5]. The performance of these filters are introduced a good results with low and middle levels of noise. But they failed with high noise's levels. 
Lately, a decision based Median Filter (DBMF) was suggested to processed the great levels of that noise by replacing pixels that recognized as noise depending on some criteria with the value that computed depending on median or the neighbor's pixels of the window. The disadvantage of this filter is the resulted image's resolution is destroyed as the level of noise is growths [6]. For that, It has been proposed the Modified Decision Based Un-symmetric Trimmed Median Filter (MDBUTMF). The work of this filter is summarized in to two steps: first distinguishing the noisy pixels, second treated using MDBUTMF. If the event that the selected window is corrupted as whole, the mean value of the window will be computed and replace it with the processed pixel value. This solution doesn't get a good alternative with high levels of noise [7].

There are many types of estimation functions. One of these functions is the Gaussian function. Gaussian function is widely used in statistics where it defines the normal distributions in many applications such as signal processing, image processing and mathematics. Gaussian functions can be described by combining the exponential function with a concave quadratic function. the Gaussian is the probability density function of a normally distributed random variable and it can be described in equation (1) in the term of exponential, mean and standard division values [8]:

$$
f=\frac{\exp \left(-\frac{(x-m)^{2}}{2 s t d^{2}}\right)}{s t d \sqrt{2 \pi}}
$$

Where $m$ denoted to mean value and std refer to standard division of the $x$ value.

This paper proposed a new method to remove noisy pixel and enhance the corrupted image by salt and pepper noise. In the proposed method a noise detection technique is used to determine the noisy pixel. Then find the size of window depending on some statistical criteria. Finally, the pixel value is estimated using one of the estimation functions.

\section{RELATED WORK}

In recent times, there are many methods to reduce Salt and Pepper noise. Rakesh M.R and et al. proposed a hybrid median filter, that developed the median filter applying cross and plus technique with $5 \times 5$ size window. Just the pixels in cross and plus directions in $5 \times 5$ window will implicate in median filter [9]. Isma I. and et al. introduced a filter with a criterion for the selection of neighboring pixel using the local and global occurrences of grey levels to predicate the pixel value [10]. T.Rajesh and et al. discussed comparison between four different image filtering methods that are Bilateral, median, ideal and Butterworth filtering, and they concluded that median filter get the best resulted image that corrupted in salt and pepper noise[11]. Others applied altered methods to remove this type of noise that corrupt the remote satellite images. Yogesh and Yogendra are applied a noise detection technique to predicated the rate of the noise in satellite images. If noise rate is less than particular threshold value, then applied the median filter. Otherwise, apply the adaptive weight algorithm. Also, they discussed the resulted satellite images with different levels of noise [12]. V.R.Vijaykumar is proposed an algorithm to remove salt and pepper noise in images based on some statistics, which used the influence function to estimate the resulted pixel. The results of different standard images with different levels of noise are illustrated [13]. Although, there are many techniques had been applied to solve the salt and pepper noise in the image, there is need to develop this methods to produce more enhancement and high quality image. In this paper, a new proposed method is introduced. That's by adapted a developed technique to noise detection and determine the size of window depending on some criteria. Then the resulted pixel is estimated using Gaussian estimation function. In the final step, the maximum filter is applied on the resulted image to get a better resolution and to improving the quality.

\section{PROPOSED METHOD}

In this paper, there is an introduced for a proposed new method to treat the particular type of noise that corrupts the image in many ways, that noise is a salt and paper noise. Figure 1 depicts the steps of the proposed method. The details of the proposed method are explained in the following steps:

Step 1 (Noise detection): For each pixel in the corrupted image a(i,j), we determined if the pixel is uncorrupted, that mean it is in the range $0<\mathrm{a}(\mathrm{i}, \mathrm{j})<255$, then return the same value of the pixel. Otherwise, go to the next step. 


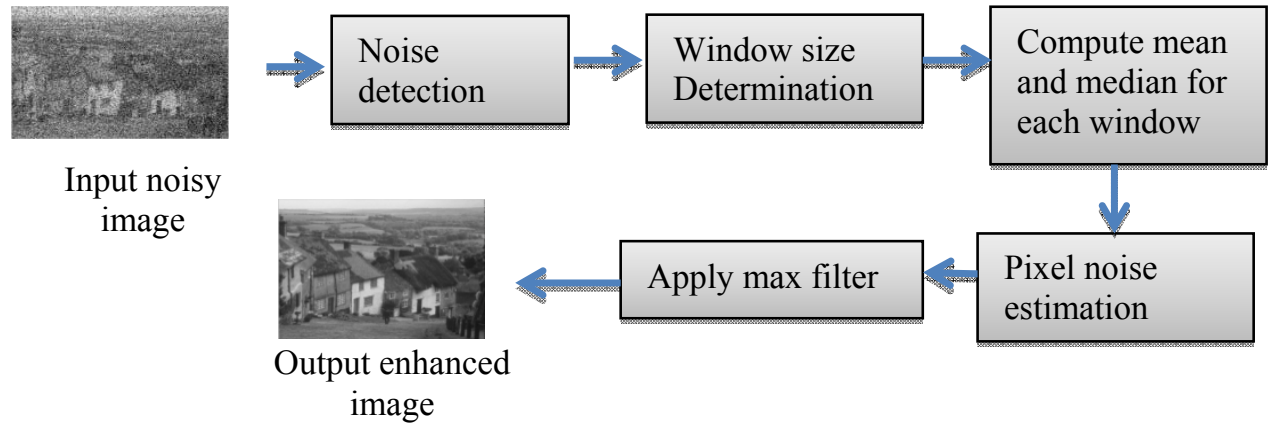

Figure 1. Proposed method processed stages

Step 2 (Window size determination): in this step, the size of the window is determined depending on the ratio of the number of the uncorrupted pixels (nupixel) in the window to the size of the whole window according to the following equation

$$
\text { ratio }=\frac{\text { nupixel }}{N \times N}
$$

Where $\mathrm{N}$ is represent the size of the current window.

In this paper, this ratio is compared with threshold (its value is fixed to 0.3 ). If the value of ratio is equal or more than threshold it will be depended. That because when the number of uncorrupted pixels is less than this ratio will be not enough to estimate the current pixel, so, the window will be described as mostly corrupted and not utilized to processed the noise. The initial size of the window will be $3 \times 3$ then increased to be $5 \times 5,7 \times 7$, and so on. The maximum size of the window will be $11 \times 11$.

Step 3 (Computation of mean and median for each window): compute the mean $(m)$ value for the window by applying equation (3) for the current pixel and find the median value $(m d)$ by sort the window's values. The median value will be the middle value in the sorted window' values.

$$
m=\frac{\sum_{i=1}^{N} \sum_{j=1}^{N} w(i, j)}{N \times N}
$$

Where $w$ denoted to the window of noisy image and $N$ denoted to the size of the current window.

Step 4 (Applying Gaussian Estimation function): For each uncorrupted pixel $p$ in the window that lies in the range $0<p<255$, do the following:

Step (4-1): Find $x$ which is represent the absolute value of the difference between the median value and the pixel as in equation (4).

$$
x=|m d-p|
$$

Step (4-2): Find t the local standard division stdloc for the current pixel.

Step (4-3): Find the Gaussian Estimated function $f$ for the current pixel, which is computed using equation (1). Where $m$ denoted to mean value, std in the equation refer to the local standard division stdloc of the pixel $p$ value and $\mathrm{x}$ denoted to the $\mathrm{x}$ value that computed in equation (4).

Step 5 (Estimate the pixel value): The estimated pixel value is computed as the ratio between $r_{1}$ and $r_{2}$ that computed as equations (5) and (6) and this value will be putted in the center of the window. The estimated pixel value is computed using equation (7).

$$
r_{1}=\sum_{i=1}^{k}\left(s(i)+\frac{f}{x}\right)
$$

And

$$
r_{2}=\sum_{i=1}^{k} \frac{f}{x}
$$

Where $k$ is represent the uncorrupted pixels number in the window. These pixels denoted as $s(i)$ in equation (5). Then the resulted pixel from this step is computed as: 


$$
B(i, j)=r 1 / r 2
$$

The steps from 1 to 5 are repeated for each pixel in the corrupted image to get the image $B$.

Step 6 (Maximum filter): Apply the max filter with window of size $3 \times 3$ on the resulted image $B$. This is done by replacing the center value of the window with the maximum value.

\section{RESULTS}

The proposed method used a resizable window depending on the amount of noise in the window, and adapted the statistic Gaussian estimation function to predicate the pixels that make this method have a good performance comparing with the other methods. The proposed method is applied on 8-bits per pixel standard gray images with the size 512 x 512, for example Goldhill, Lena and Boat. The quality of the resulted images is measured using PSNR and SSIM quality measurements that illustrated in 8 and 9 equations respectively.

$$
P S N R=10 \log _{10}\left[\frac{255^{2}}{\frac{1}{M N} \sum_{i} \sum_{j}\left(y_{i j}-x_{i j}\right)^{2}}\right]
$$

Where the value $y_{i j}$ denoted to the reconstructed image pixels and the value $x_{i j}$ denoted to the original image value. $\mathrm{Mx} \mathrm{N}$ is the size of image

$$
\operatorname{SSIM}(x, y)=\left(\frac{2 \mu_{x} \mu_{y}+d_{1}}{\mu_{x}^{2}+\mu_{y}^{2}+d_{1}}\right) \times\left(\frac{2 \sigma_{x y}+d_{2}}{\sigma_{x}^{2}+\sigma_{y}^{2}+d_{2}}\right)
$$

Where $\mathrm{x}$ and $\mathrm{y}$ are the windows of $\mathrm{X}$ and $\mathrm{Y}$ images respectively. $\mu \mathrm{x}$ and $\mu \mathrm{y}$ are represented the values of mean for the windows $\mathrm{x}$ and $\mathrm{y}$ respectively. $\sigma_{\mathrm{x}}, \sigma_{\mathrm{y}}$ are denoted to the $\mathrm{x}$ and $\mathrm{y}$ standard deviation respectively. $\sigma_{\mathrm{xy}}$ refer to the cross-correlation of the mean shifted images $\mathrm{x}-\mu \mathrm{x}$ and $\mathrm{y}-\mu \mathrm{y}$, and the di for $\mathrm{i}$ $=1,2$ are small positive constants. These constants avoid divided by zero issues when either $\left(\mu_{\mathrm{x}}^{2}+\mu_{\mathrm{y}}^{2}\right)$, $\left(\sigma_{\mathrm{x}}^{2}+\sigma_{\mathrm{y}}^{2}\right)$ or $\left(\sigma_{\mathrm{x}} \sigma_{\mathrm{y}}\right)$ is close to zero. The global SSIM quality image that refer to the images $\mathrm{x}$ and $\mathrm{y}$ can be calculated by averaging the SSIM values computed for small spatial windows of the two images.

Figures (2), (3) and (4) illustrated the proposed method's effect on Goldhill, Boat and Lena images that have salt and pepper noise with rate $40 \%, 60 \%$ and $90 \%$ respectively.

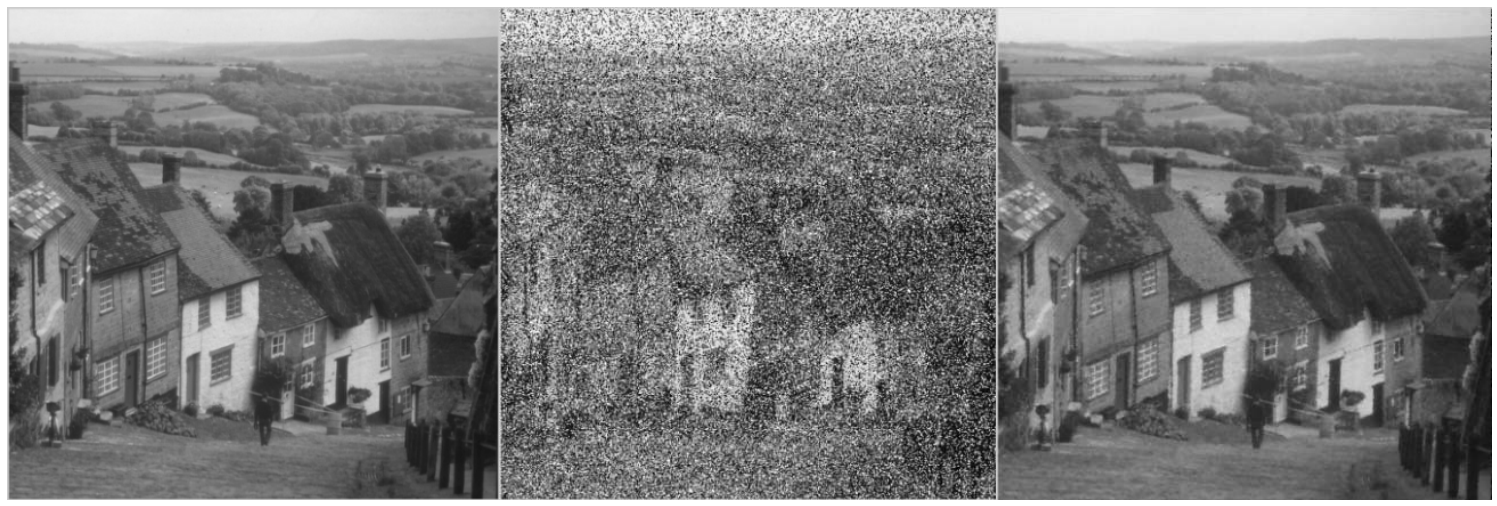

(a) Original Goldhill image

(b) distorted Goldhill image

(c) Treated image

Figure 2. Proposed Method effect on Goldhill image 40\% Salt and pepper noise 


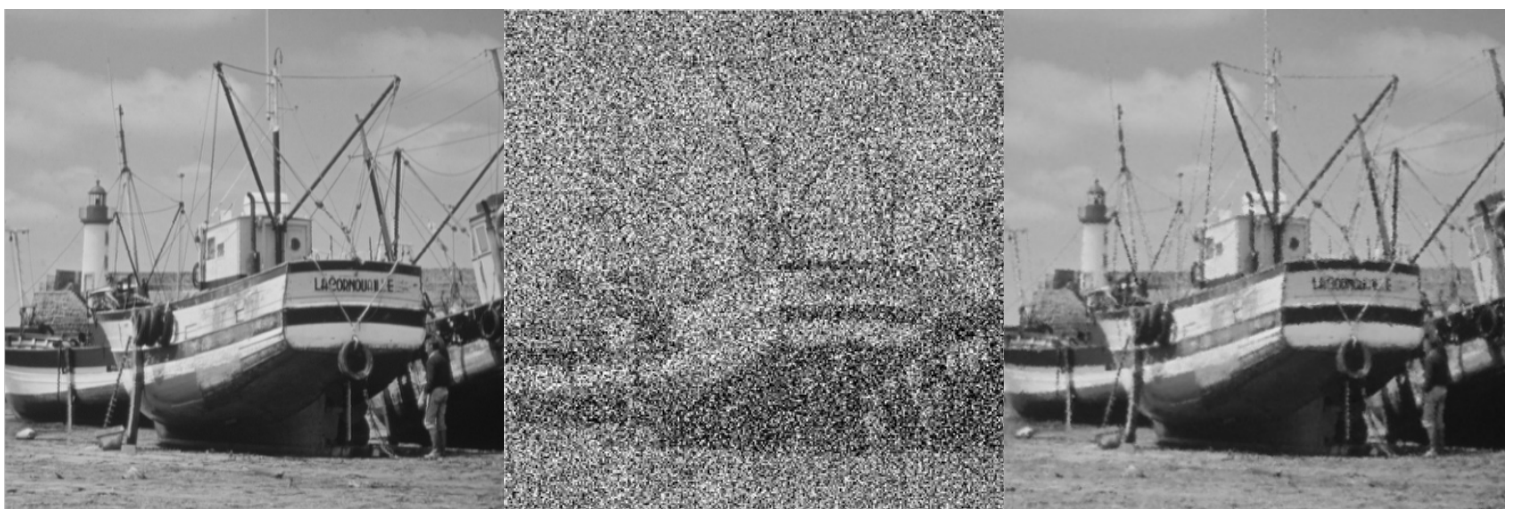

(a) Original Boat image

(b) distorted Boat image

(c) Treated image

Figure 3. Proposed Method effect on Boat image $60 \%$ salt and pepper noise

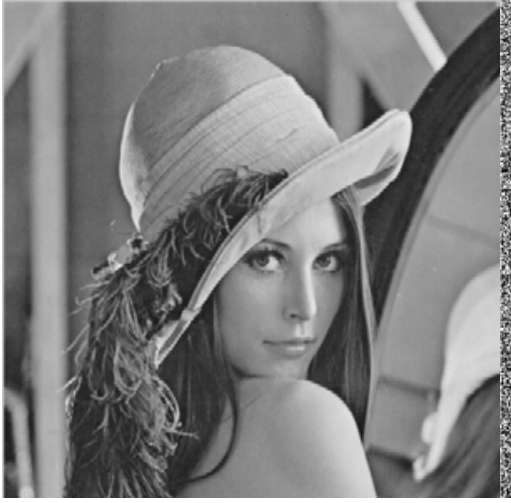

(a) Original Lena image

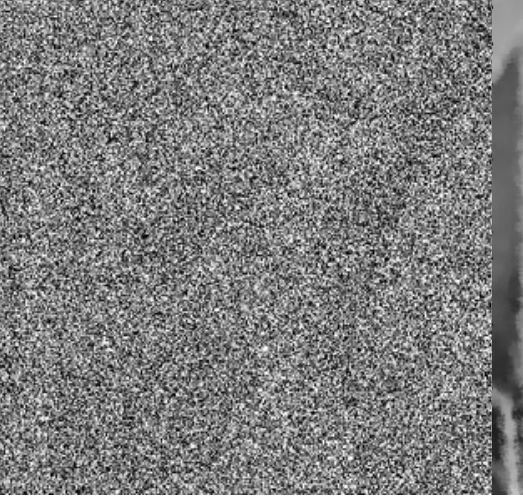

(b) distorted Lena image

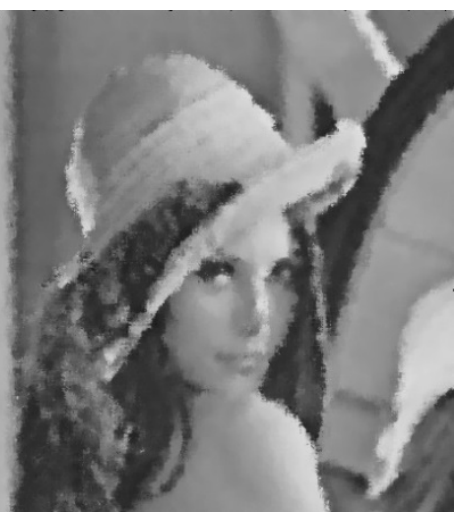

(c) Treated image

Figure 4. Proposed Method effect on Lena image 90\% Salt and pepper noise

Table 1 shows SSIM and PSNR results for Goldhill, Boat and Lena images with different salt and pepper's level noise by applying our proposed method.

To show the efficiency of our proposed method, the proposed algorithm (PA) is compared with various algorithms like Standard median filter (SMF), Adaptive median filter (AMF), Progressive switching median filter (PSMF), Decision based median algorithm (DBMF), and Modified Decision Based Unsymmetrical Trimmed Median Filter (MDBUT), as computed in [7]. The comparison is done using PSNR measure as shown in Table 2. These results are computed for Lena standard image for different levels of salt and pepper noise.

Table 1. Illustration of SSIM and PSNR for corrupted images with different levels of noise

\begin{tabular}{ccccccc}
\hline \multirow{2}{*}{ Noise Levels } & \multicolumn{2}{c}{ Goldhill } & \multicolumn{3}{c}{ Boat } & \multicolumn{2}{c}{ Lena } \\
& SSIM & PSNR & SSIM & PSNR & SSIM & PSNR \\
\hline $10 \%$ & 0.984 & 39.217 & 0.987 & 38.552 & 0.992 & 31.239 \\
$20 \%$ & 0.966 & 36.146 & 0.976 & 35.624 & 0.984 & 38.702 \\
$30 \%$ & 0.946 & 34.135 & 0.963 & 33.599 & 0.974 & 36.355 \\
$40 \%$ & 0.922 & 32.516 & 0.946 & 31.862 & 0.961 & 34.260 \\
$50 \%$ & 0.894 & 31.086 & 0.926 & 30.169 & 0.945 & 32.607 \\
$60 \%$ & 0.856 & 29.573 & 0.896 & 28.455 & 0.918 & 30.515 \\
$70 \%$ & 0.794 & 27.603 & 0.844 & 25.932 & 0.874 & 28.111 \\
$80 \%$ & 0.708 & 25.733 & 0.77 & 23.728 & 0.799 & 25.404 \\
$90 \%$ & 0.626 & 24.388 & 0.691 & 22.194 & 0.731 & 23.63 \\
\hline
\end{tabular}


Table 2. Comparison in PSNR between the proposed algorithm and other methods

\begin{tabular}{|c|c|c|c|c|c|c|}
\hline Noise Levels & SMF & AMF & PSMF & DBMF & MDBUTME & PA \\
\hline $10 \%$ & 28.49 & 36.30 & 30.86 & 36.98 & 36.67 & 41.239 \\
\hline $20 \%$ & 25.75 & 29.20 & 28.28 & 33.22 & 32.65 & 38.702 \\
\hline $30 \%$ & 21.85 & 23.72 & 25.26 & 30.38 & 30.19 & 36.355 \\
\hline $40 \%$ & 18.41 & 18.60 & 22.36 & 28.23 & 28.32 & 34.260 \\
\hline $50 \%$ & 14.73 & 15.33 & 19.18 & 26.49 & 26.62 & 32.607 \\
\hline $60 \%$ & 12.23 & 12.20 & 12.15 & 24.72 & 24.73 & 30.515 \\
\hline $70 \%$ & 9.98 & 9.95 & 9.76 & 22.66 & 22.38 & 28.111 \\
\hline $80 \%$ & 8.02 & 8.26 & 8.09 & 20.42 & 20.07 & 25.404 \\
\hline $90 \%$ & 6.58 & 6.65 & 6.62 & 17.23 & 17.39 & 23.63 \\
\hline
\end{tabular}

\section{CONCLUSION}

This paper introduced a developed algorithm to enhance the corrupted image with salt and pepper noise. This method introduced a good results compared with the other algorithms. This method used a resizable window schema to get the appropriated size of window that have a number of uncorrupted pixels that important in estimation process. The using of Gaussian estimation function in statistical method led to a good quality and image enhancement. The PSNR metric illustrated in Table 1 ranged from 23 to 41 along with different noise levels. Although, the high difference in noise level, the SSIM metric have a slight changing and have an accepted results with high levels of noise like $80 \%$ and $90 \%$. In the other words, the good results of SSIM metric denoted that the proposed method have a good performance and a visual quality, where SSIM motivated by the observation that natural images have highly structured signals with strong neighborhood dependencies. These dependencies carry useful information about the structures of the objects in the visual scene.

\section{REFERENCES}

[1] R. N. Kulkarni and P. C. Bhaskar, "Decision Based Median Filter algorithm using Resource Optimized FPGA to Extract Impulse Noise," International Journal of Reconfigurable and Embedded Systems (IJRES), vol/issue: 3(1), 2014.

[2] Hung T. S., et al., "Fast two dimensional median filtering algorithm," IEEE Transactions on Acoustics Speech and Signal Processing, vol. 1, pp. 13-18, 1979.

[3] Arce G. and Paredes J., "Recursive Weighted Median Filters Admitting Negative Weights and Their Optimization," IEEE Trans. on Signal Processing, vol/issue: 48(3), pp. 768-779, 2000.

[4] Hwang H. and Haddad R. A., "Adaptive median filters: new and results," IEEE Trans. on Image Processing, vol/issue: 4(4), pp. 499-502, 1995.

[5] Z. Wang and D. Zhang, "Progressive switching median filter for the removal of impulse noise from highly corrupted images," IEEE Transactions on Circuits and Systems II, vol. 46, pp. 78-80, 1999.

[6] Srinivasan K. S. and Ebenezer D., "A New Fast and Efficient Decision-Based Algorithm for Removal of HighDensity Impulse Noises,” IEEE signal processing letters, vol/issue: 14(3), pp. 189 -192, 2007.

[7] M. Anand and Y. Narasimha, "Removal of Salt and Pepper Noise from highly Corrupted Images using Mean Deviation statistical parameter," International Journal on Computer Science and Engineering (IJCSE), vol/issue: 5(02), 2013.

[8] Wikipedia encyclopedia, https://en.wikipedia.org/wiki/Gaussian_function.

[9] Rakesh M. R, et al., "Hybrid Median Filter for Impulse Noise Removal of an Image in Image Restoration," International Journal of Advanced Research in Electrical, Electronics and Instrumentation Engineering, vol/issue: 2(10), 2013

[10] Isma I., et al., "Salt and Pepper Noise Removal Filter for 8-Bit Images Based on Local and Global Occurrences of Grey Levels as Selection Indicator," Nepal Journal of Science and Technology, vol/issue: 15(2), pp. 123-132, 2014.

[11] T. Rajesh, et al., "An Efficient Image Denoising Technique for Various Noisy Images," International Journal Of Engineering And Computer Science, vol. 3, pp. 5064-5066, 2014.

[12] Yogesh V. and Yogendra K., "Removal of Salt and Pepper Noise from Satellite Images," International Journal of Engineering Research \& Technology (IJERT), vol. 2, pp. 2051-2058, 2013.

[13] V. R. Vijaykumar, et al., "Robust Statistics Based Algorithm to Remove Salt and Pepper Noise in Images," International Journal of Information and Communication Engineering, 2009. 